\title{
New Approaches to Modeling and Analysis of Sleep Apnea Events Data from Infants Using Pacifiers for Improved Diagnosis of Obstructive Sleep Apnea
}

\author{
Sujay Datta ${ }^{1}$ \\ ${ }^{1}$ University of Akron \\ Dept. of Statistics, 302 Buchtel Common, Akron, OH 44325, USA \\ sd85@uakron.edu
}

\section{Extended Abstract}

Polysomnography is an overnight systematic procedure to collect physiological parameters during sleep. It is considered as gold-standard for diagnosing sleep-related disorders. It takes several days to score and interpret the raw data from this study and confirm a diagnosis of, say, Obstructive Sleep Apnea (OSA) - a potentially dangerous disorder. The presence of artifacts (anomalies created by a malfunctioning sensor) makes scoring even more difficult, potentially resulting in misdiagnosis. It is common to see airflow signal artifacts in infants that use a pacifier during sleep. The act of sucking on the pacifier causes artifacts in the oro-nasal sensor (thermistor) used to monitor airflow during respiration. The resulting inaccurate scoring leads to an under-estimation of the Apnea Hypopnea Index (AHI) -- the basis for a formal OSA diagnosis. So researchers are now exploring two other information sources (blood oxygen saturation readings from a pulse-oximeter and occurrence of arousal events) to supplement the artifact-corrupted thermistor data. They first look for statistical association between the thermistor and the pulse-oximeter/arousal data and then statistically predict a modified AHI score using the latter whenever the former is corrupt. To our knowledge, no attempt of statistically modeling these three data-sources to bring out their association currently exists. This project aims at developing several competing probabilistic models for these data-types and comparing them using archived data from the Akron Children's Hospital.

After performing some statistical tests for association, first a simple Bayesian posterior probability calculation is done using the Bayes formula. The posterior probability of an apnea/hypopnea event given that either a dip in the Pulse Oximeter oxygen saturation value or an arousal event or both has (have) occurred is calculated. The posterior expectation calculated using this posterior probability will help us adjust for the undercount of apnea-hypopnea events, thereby adjusting the AHI for better accuracy. Subsequently, in the Beta-Binomial model, the occurrence or non-occurrence of an apnea/hypopnea event will be modeled using a Binomial distribution and its parameter will be assumed to have a Beta prior distribution whose parameters (called the hyper-parameters) will be estimated from the data or subjectively chosen. Once again, the posterior expectation calculated using this posterior probability will help us adjust for the underestimation of AHI.

We have also tried some novel approaches. One such approach is to think of each sequence of events as the sequence of state-changes in a discrete homogeneous Markov chain (the other states in those three Markov chains being a normal breath-cycle, a normal Pulse Ox reading and a non-arousal). Then the question is what can we infer about the "normal to apnea/hypopnea' transition probabilities of the first Markov chain from the state transition probabilities of the other two Markov chains using the theory of double chain Markov models (DCMM). Another equally novel approach is to model the three sequences of Thermistor readings, Pulse Ox readings and EEG each as a homogeneous Poisson process. Then to infer about events in the first Poisson process, given observed events in the other two processes, one will use the theory of correlated Poisson processes.

\section{References}

[1] Rundo, J. V., \& Downey, R. (2019). Chapter 25-Polysomnography. In K. H. Levin \& P. Chauvel (Eds.), Handbook of Clinical Neurology (pp. 381-392). https://doi.org/10.1016/B978-0-444-64032-1.00025-4

[2] Adair, S.M., "Pacifier Use in Children: A Review of Recent Literature," Pediatr Dent. 2003:25:449-458. 
[3] Lau, Chantal. "Development of Suck and Swallow Mechanism in Infants." National Institute of Health. 2015. DOI 10.1159/000381361.

[4] MacLean, Joanna E., et al, "Developmental Changes in Sleep and Breathing across Infancy and Childhood." Paediatric Respiratory Reviews. 2015. DOI 10.1016/j.prrv.2015.08.022.

[5] Oder, A.L, et al, "Short-Term Effects of Pacifier Texture on NNS in Neurotypical Infants," International Journal of Pediatrics. 2013. DOI 10.1155/2013/168459.

[6] Liebl, D. \& Rameseder, S. (2018). "Partially Observed Functional Data: The Case of Systematically Missing Parts". arXiv: $1711.07715 \mathrm{v} 3$ [stat.ME] 30 July 2018

[7] Berchtold, A. (1999). "The Double-Chain Markov Model”. Tech. report no. 348, Dept. of Statistics, University of Washington, Seattle, USA 